\title{
Kerr Black Hole Geometry Leading to Dark Matter and Dark Energy via E-Infinity Theory and the Possibility of a Nano Spacetime Singularities Reactor*
}

\author{
Mohamed S. El Naschie \\ Department of Physics, University of Alexandria, Alexandria, Egypt \\ Email: chaossf@aol.com
}

Received 18 March 2015; accepted 21 April 2015; published 23 April 2015

Copyright (C) 2015 by author and Scientific Research Publishing Inc. This work is licensed under the Creative Commons Attribution International License (CC BY). http://creativecommons.org/licenses/by/4.0/

(c) (;) Open Access

\section{Abstract}

The present paper is basically a synthesis resulting from incorporating Kerr spinning black hole geometry into E-infinity topology, then letting the result bares on the vacuum zero point Casimir effect as well as the cosmic dark energy and dark matter density. In E-infinity theory a quantum particle is represented by a Hausdorff dimension $\phi$ where $\phi=2 /(\sqrt{5}+1)$. The quantum wave on the other hand is represented by $\phi^{2}$. To be wave and a particle simultaneously intersection theory leads us to $(\phi)(\phi)^{2}=\phi^{3}$ which will be shown here to be twice the value of the famous Casimir force of the vacuum for a massless scalar field. Thus in the present work a basically topological interpretation of the Casimir effect is given as a natural intrinsic property of the geometrical topological structure of the quantum-Cantorian micro spacetime. This new interpretation compliments the earlier conventional interpretation as vacuum fluctuation or as a Schwinger source and links the Casimir energy to the so called missing dark energy density of the cosmos. From the view point of the present work Casimir pressure is a local effect acting on the Casimir plates constituting the local boundary condition while dark energy is nothing but the global combined effect of infinitely many quantum waves acting on the Möbius-like boundary of the holographic boundary of the entire universe. Since this higher dimensional Möbius-like boundary is one sided, there is no outside to balance the internal collective Casimir pressure which then manifests itself as the force behind cosmic expansion, that is to say, dark energy. Thus analogous to the exact irrational value of ordinary energy density of spacetime $E(O)=\left(\phi^{5} / 2\right) m c^{2}$ we now have $P($ Casimir $)=\left(\phi^{3} / 2\right)\left(c \hbar / d^{2}\right)$ where $c$ is the speed of light, $m$ is the mass, $\hbar$ is the Planck constant and $d$ is the plate sepa-

\footnotetext{
"Dedicated to all those who genuinely believe in the science and reality of zero point spacetime vacuum energy.
} 
ration. In addition the new emerging geometry combined with the topology of E-infinity theory leads directly to identifying dark matter with the quasi matter of the ergosphere. As a direct consequence of this new insight $E=m c^{2}$ which can be written as $E=E(O)+E(D)$ where the exact rational approximation $E(O)=m c^{2} / 22$ is the ordinary energy density of the cosmos and the exact rational approximation $E(D)=m c^{2}(21 / 22)$ is the corresponding dark energy which could be subdivided once more albeit truly approximately into $E(D)=m c^{2}(5 / 22)+m c^{2}(16 / 22)$ where 5 is the Kaluza Klein spacetime dimension, 16 are the bosonic extra dimensions of Heterotic superstrings and $5 / 22 \simeq 22 \%$ is approximately the density of the dark matter-like energy of the ergosphere of the Kerr geometry. As for the actual design of our nano reactor, this is closely related to branching clusters of polymer, frequently called lattice animals. In other words we will have Casimir spheres instead of Casimir plates and these spheres will be basically nano particles modelling lattice animals. Here $D=4$ will be regarded as spacetime dimensionality while $D=6$ of percolations are the compactified super string dimensions and $D=8$ is the dimension of a corresponding super space.

\section{Keywords}

Casimir Effect, Dark Energy, E-Infinity, Cantorian Spacetime, Kerr Spacetime Geometry, Energy from the Ergosphere, Spinning Black Holes, Nano Spacetime Reactors, Kaluza-Klein Spacetime, Heterotic Superstrings

\section{Introduction}

The present work has three different aims to achieve and three different messages to communicate. The scientific part is centred around the quantum vacuum and its singularities as a source of energy [1]-[58]. The rest is centred around a socio-economical, political message and aim, namely that we must invest in this new revolutionary source of energy [59]-[61]. The idea of zero point energy and the fluctuation of vacuum may seem at first glance to be more science fiction than science fact [62]-[80]. However there are, and since quite some time, a host of hard core experimental evidence that the vacuum may be more real and fundamental than most of what we habitually consider the materialistic reality of physical phenomena [1]-[58]. We just need to mention in this context the Lamb shift, Schwinger correction [62]-[66] and the van der Waals forces to realize how physical and real the vacuum is [22]-[24] [28]. None the less, and we do not think it is a minority opinion, nothing could be more impressive and inspiring as the Casimir effect (see Figure 1) [22]-[24]. This effect is a natural consequence and fundamental aspect of quantum field theory. There are at least two fundamental interpretations of this miraculous effect [63] [64]. The first is loosely connected to boundary conditions and the zero point quantum vacuum fluctuation which may be the common way of looking at the Casimir effect within the working physicists community. The second, which may be more theoretical and fundamental, is to see Casimir as a source in the mould of J. Schwinger's way of thinking and not far from the Casimir operators of quantum field theory [62] [63] [66]. Thus we could look upon the Casimir effect as a cousin of Hawking's negative energy fluctuation around a black hole or as Unruh's temperature for an accelerated, observed in a Rindler wedge, universe. Alternatively we could follow Schwinger's ideas and see it as something related to a fundamental mathematical scenario such as the Banach-Tarski theorem advanced for the first time in the cosmology of the big bang by the present author [67] [68].

Unlike in previous publications, in the present work we rely heavily on Kerr spacetime geometry of rotating black holes (see Figure 1) [70] [71]. This is of course not experimentally verified like the Casimir energy nor even like the conjectured dark energy. Never the less, Kerr geometry and its ergosphere [70] [71] ties in almost perfectly with our E-infinity dark energy theory so that we could regard it as a candidate for experimental discovery sooner than many may expect.

In the present analysis however, and as far as the Casimir effect is concerned, we opted for a rather different way of viewing the Casimir effect as a natural topological necessity of a Cantorian spacetime fabric that was 


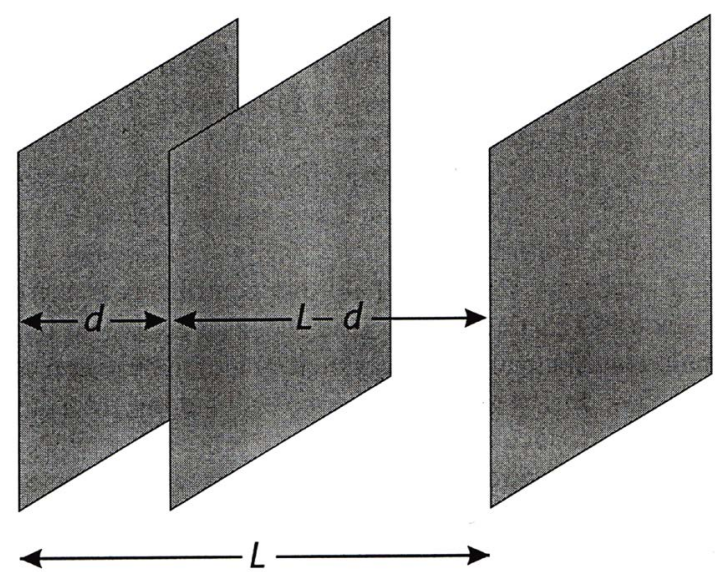

Figure 1. A Casimir effect gedanken experiment with three rather than two perfectly conducting uncharged plates. Some work is currently underway by the author and his group to replace the flat plates by a mixture of spheres and flat plates and then proceed from there to use an uncharged swarm of Fullerene particles and a variety of other nano particles then extract the $96 \%$ energy near to the surface of this fractal-like Bethe lattice [77]-[80].

woven from an infinite number of zero Cantor sets and empty Cantor sets [37]. The zero set is taken following von Neumann-Connes dimensional function [69] to model the quantum particles [52] [56] while the empty set models the quantum wave [56]. Following this road we come to realize that the Casimir latent energy is nothing but the universal fluctuation $\phi^{3}$ which gives birth to the core of Cantorian-fractal spacetime by inversion $1 / \phi^{3}=4+\phi^{3}=4.23606797$ where $\phi=(\sqrt{5}-1) / 2 \quad$ [52] [53]. For a massless scalar field this is nothing but the difference between the Hausdorff dimension of the particle zero set $\phi$ and the wave empty set $\phi^{2}$ producing the topological pressure behind the Casimir physical pressure. The result not surprisingly is almost equal to double the value found using imaginative modification of the classical Casimir experiment by Zee shown in Figure 1 [62] who found the dimensionless Casimir energy for a massless scalar field to be $\pi / 24 \simeq 0.1308$ [62]. Using E-infinity methodological reasoning, the exact value of Zee in the limit must be the ratio of the dimensionality of a Calabi-Yau transfinite manifold $6+k=6.18033889$ and the transfinite dimension of bosonic string theory, i.e. 26.18033989 [50] [53]-[56]. That means $(6+k) /(26+k)=\phi^{3} / 2$ Needless to say, the division by 2 is due to the subdivision of the "vacuum" of E-infinity theory and is analogous to dividing Hardy's entanglement $P(H)=\phi^{5}$ by 2 to obtain the density of the ordinary measurable energy of the cosmos $E(O)=\left(\phi^{5} / 2\right) m c^{2}$ [53]-[56]. The dimensional quantity analogous to $m c^{2}$ for the Casimir effect is trivially clear to be $\hbar c$ where $c$ is the speed of light and $\hbar$ is the Planck quantum. From this new topological interpretation it becomes obvious that Casimir $\phi^{3}$ is the counterfactual or global part of Hardy's entanglement $P(H)=\left(\phi^{3}\right)\left(\phi^{n}\right)$ where $\mathrm{n}$ is the number of quantum particles and is found for $n=0$. It is therefore closely related to the Unruh temperature where $n=1$, the Immirzi parameter $n=3$ as well as Hardy's generic quantum-topological entanglement $n=2$. In fact Unruh temperature is a dynamical Casimir effect and can be found by giving $\left(\phi^{3} / 2\right)$ a spin structure similar to spacetime Hausdorff dimension $5+\phi^{3}$ obtained from multiplying $4+\phi^{3}$ with $1+\phi^{3}$. These insights are not only simple mathematical insights. It goes far beyond that and suggests that Casimir energy and dark energy are two sides of the same coin, differing only with regard to exo and endo boundary conditions [6] [7] which will be made clearer in the main body of the present work. Second, by manipulating the local dimensionality of spacetime using an elaborate and complex set up of Casimir plates system representing at the end a multi-dimensional fractal sphere [9] we could build a nano universe in principle and extract its dark energy concentrated at its boundary. The way to do this economically may be five, ten or more years ahead of experimental work using the modern developments of cutting edge nanotechnology [1] [73]. Never the less, the promise of near to infinite, clean, free energy is a goal worth any effort and the financial risks are minimal compared to the possible gains, so let the present modest steps be the first into this new world of a nano, Casimir-dark or ordinary energy reactor. To obtain real energy from the topology of spacetime must strike 
a down to earth engineer like the present author who grew up in the realistic rigor of theory of elasticity, Koiter theory of buckling instability and engineering science as a wonder which is never the less no wonder because it is spacetime which gives rise to matter and not the other way around as our primitive human senses lead us wrongly to believe not by mathematical logic but by pure habits and the self centeredness and bias of a materialistic creature looking at creation from his limited materialistic viewpoint [75].

We can improve our model considerably by drawing into the preceding picture the possibilities offered by the Kerr spacetime geometry in Figure 2 [70] [71]. Thus $E=m c^{2}$ can be subdivided not only into the two well known parts $E(O)=m c^{2} / 22$ for the ordinary measurable energy density of the cosmos and $E(D)=m c^{2}(21 / 22)$ for the corresponding dark energy density but $E(D)$ dark energy itself could be subdivided, albeit approximately, into $E_{3}=m c^{2}(5 / 22)$ for dark matter and $E_{4}=m c^{2}(16 / 22)$ for pure dark energy. The reader may have already surmised that $E_{3} \simeq 22 \%$ is dark matter energy stored in the ergosphere of the Kerr spinning singularity while 5 is the Kaluza-Klein spacetime dimension and 16 is the bosonic extra dimension of Heterotic superstring theory so that $5 / 22 \simeq 22 \%$ is the Lorentzian factor for dark matter-like energy and $5+16=21$ are the total compactified dimensions. That way we have $2621=5$ Kaluza-Klein dimensions as should be. The relation between $D=5$ and $D=4$ is well known and will not be discussed here [46] [52]. However our drive towards a physical realization of our Casimir nano reactor theory will make extensive use of the obvious analogy between branching polymers, Bethe lattice and Cantorian fractal spacetime topology (see Figure 3).

\section{The Basic Principles of a Casimir Reactor}

We give here a general outline of the theoretical principle and basic design concepts of a proposed Casimir dark energy nano reactor [1]. In a nutshell the theory and consequently our actual design depends crucially upon the equivalence between the dark energy density of the cosmos and the faint local Casimir effect produced by two sides boundary condition quantum waves [1]-[8]. This similarity is true because both types of energy are linked to the quantum wave although dark energy is repulsive while Casimir energy is like ordinary energy of the quantum particle, i.e. attractive. This Casimir effect is then colossally amplified as a one-sided quantum wave pushing from the inside with nothing balancing it from the non-existent outside [1]. That is the morphogenesis by which Casimir attractive forces transform into circulatory repulsive dark energy at the one sided Möbius-like boundary of the holographic boundary of the universe located at the hyperbolic infinity. In view of the present theory, this is essentially what led to the observed accelerated expansion of the cosmos. As in any reactor, the basic principle in the present design is to produce a gradient so that the excess energy on one side flows to the other side. Thus in principle we will restructure the local topology of space using material nanoscience technology to create an artificial local high dimensionality with a Dvoretzky theorem like volume measure concentration [2] [3]. This may sound far more difficult than it is really because we know from diffusion limited aggregation and clustering on fractals that the dimensionality of the real processes can be substantially higher than the dimensionality of the $3+1$ embedding spacetime. Without going into the intricate nonlinear dynamics and technological detail, it is fair to say that this would lead us to pure, clean, free energy obtained directly from the topology of spacetime and to stress this aspect we give an extensive list of references touching upon this point from a multitude of different viewpoints [1]-[69]. Needless to say the entire design is based completely on the theory of quantum wave dark energy proposed by the present author for the first time in 2011 in a conference held in the Bibliotheca Alexandrina, Egypt and a little later in Shanghai, Republic of China [50] [53]. The quintessence of the present theory is easily explained as the $\phi^{3}$ latent Casimir topological pressure energy where $\phi=(\sqrt{5}-1) / 2$ produced from the zero set $\phi$ of the quantum particle when we extract the empty set quantum wave $\phi^{2}$ from it and find $\phi-\phi^{2}=\phi^{3}$ by restructuring space via conducting but uncharged plates similar to that of the classical Casimir experiments but with some modification. In other words by inducing a topological change in quantum spacetime we obtain energy in classically impossible ways, which is what the Casimir force of a massless scalar field $\phi^{3} / 2$ is all about. Said in a different way, between the plates we have the empty set topological pressure $\phi^{2}$ while outside we have the zero set pressure $\phi$ and that gives us the attractive net pressure $\phi^{2}-\phi=-\phi^{3}$ with the right negative sign convention.

\section{Analysis of a Casimir Gedanken Experiment}

It is an almost trivial matter to visualize and understand intuitively the physics behind the Casimir effect if we 


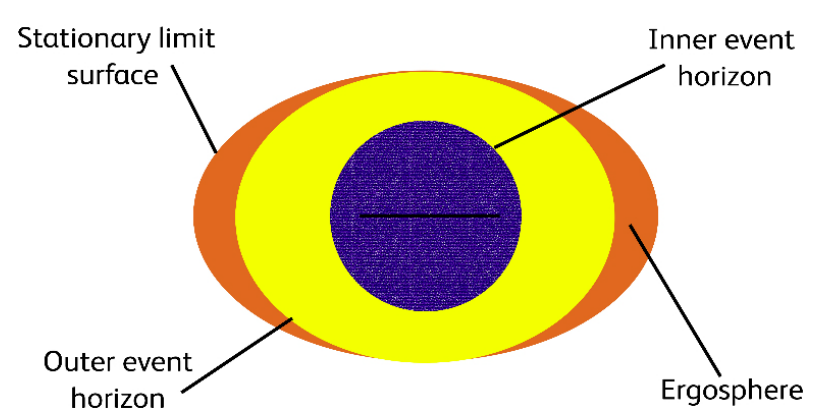

Figure 2. The spinning Kerr black hole with its two event horizons. In the circular singularity we have the $4.5 \%$ ordinary energy, the remaining $95.5 \%$ is divided into pure dark energy and pure dark matter-like energy in the ergosphere. It is generally accepted that a Planck mass constitutes a micro black hole. However in view of Kerr's spinning black hole and the resent results, we could push the idea further and claim that all pre-elementary particles are one of two kinds of black holes. They are either what later will become bosons and these are basically non-rotating black holes in origin while rotating Kerr black holes are what basically will become fermions. We remind the reader that the Higgs boson has a zero spin but there is no fermion in reality or theory that has a zero spin. That is an additional reason to look at the ergosphere as the "home" of dark matter, having "spin" inherited from the geometry of Kerr spacetime singularity. The end result is a triadically dissected $E$, namely $E=\left(m c^{2} / 22\right)+m c^{2}(16 / 22)+m c^{2}(5 / 22)$ where the first term is the ordinary energy, the second is the dark energy and the third is the dark matter density.

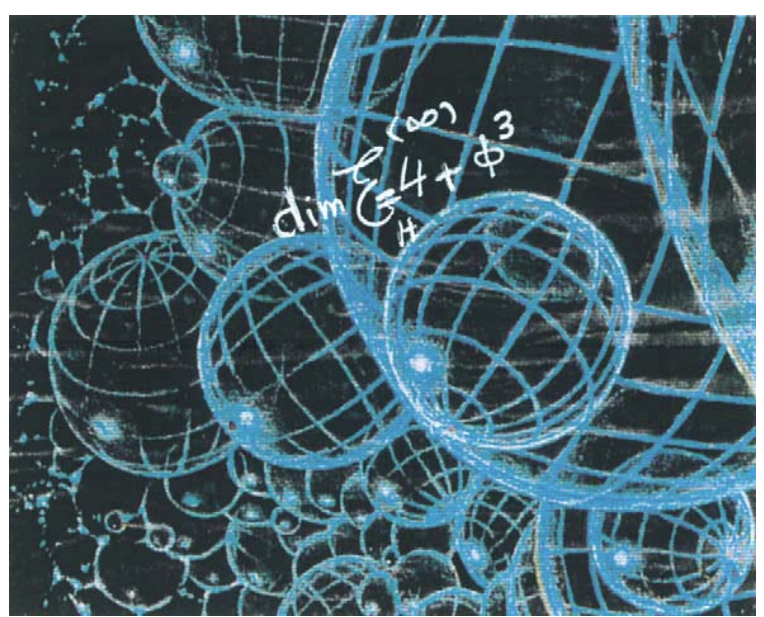

Figure 3. Turbulent Apollonian fractals with spheres of all dimensionalities from zero to infinity on all scales as a model for E-infinity space or a kind of polymerized spherical micro spacetime [78] [79] with a formal dimension equal to infinity, an expectation value for the Hausdorff dimension equal to $4+\phi^{3} \simeq 4.236067977$ and an expectation value for the topological dimension equal to exactly 4 . In other words this is the E-infinity spacetime. Seen that way we could replace these spheres with Bucky nano particles of all combinations and produce an artificial E-infinity-like spacetime-Casimir nano reactor [73] [78] [79].

replace in our mind quantum waves and quantum spacetime by the mental picture of real sea waves. Imagining two large walls brought just a meter apart and placed in relatively shallow water, then clearly the two walls are more likely to be pushed together by the outside waves because the water between the walls is obviously much calmer than in the open se water outside. In other words, the Casimir effect is not a mystery. The real mystery is in the quantum wave and quantum spacetime is the solution. In Figure 1 we present a gedanken experiment to show that the net Casimir topological force is equal to half the universal global E-infinity quantum spacetime fluctuation, i.e. $\phi^{3} / 2$ where $\phi=1 /(\sqrt{5}+1)$ is the Hausdorff dimension of the zero set modelling the quantum particle. Rather than cluttering Figure 1 of the schematic Casimir set up with many labels and notations we decided to leave it bare and explain it meticulously in this section. The reader should, in the back of his mind, number the above three perfectly conducting but uncharged Casimir plates in Figure 1 as one to three, from left to right. At the two extremities the topological force-pressure is identified here with the Hausdorff dimension of 
the zero set, i.e. $\phi$ of the quantum particle. Inside however, i.e. between plates 1 and 2 as well as 2 and 3, we have the empty set with the Hausdorff dimension $\phi^{2}$, which models the quantum wave. Consequently, following this "topological" conception there is a net external topological force acting from outside on the inside equal to $\phi-\phi^{2}=\phi^{3}$. This is not the force per single plate but the intrinsic fluctuation pressure $\phi^{3}$, which is the inverse of the Hausdorff dimension of the E-infinity quantum spacetime in which the entire gedanken experiment is taking place and where the spacetime topological dimension is exactly 4 while the corresponding well known value of the Hausdorff dimension is $1 / \phi^{3}=4+\phi^{3}=4.23606799$. Seen that way the Casimir topological force is given by $\phi^{3} / 2=(1+k) / 10$ where $k=\phi^{3}\left(1-\phi^{3}\right)$. This corresponds to a dimensional value of $P($ Casimir $)=\left(\phi^{3} / 2\right)\left(\hbar c / d^{2}\right)$ where $c$ is the speed of light and $\hbar$ is the Planck constant. This is rather close to the approximate value found by A. Zee for the same arrangement assuming a massless scalar field rather than an electromagnetic field, namely $(\pi c \hbar) /\left(24 d^{2}\right)$ as given on page 66 of his classical text book "Quantum Field Theory In A Nutshell” [62]. Let us stress once more a basic topological insight of this gedanken experiment with physical deep meaning. If $\phi$ represents the particle aspect of the quantum while $\phi^{2}$ is the corresponding representation of the wave aspect, then by intersection theory $(\phi)(\phi)^{2}=\phi^{3}$ represents both simultaneously. This means physical Casimir energy is a spacetime pure topological energy. Since the Davies-Unruh temperature is nothing but a dynamical Casimir effect then by the same token it is an effect produced by the random fractal topology of spacetime. Like all Cantorian fractals, this spacetime is naturally quantized and has an in built renormalization coupled to a transfinite, i.e. infinite capacity cellular automata processing infinite details in an exact manner due to the infinite computational efficiency of its golden mean binary number system. With such an almost perfect theoretical framework it would seem to us realistic to think that all that we still need is advanced nanotechnology to be able to build a real world economically efficient Casimir reactor. Needless to say that for an electromagnetic field replacing the massless scalar field the force per unit area changes from $(\pi c \hbar) /\left(24 d^{2}\right)$ to $\left(\pi^{2} c \hbar\right) /\left(240 d^{4}\right)$ [62]. The E-infinity derivation in this case will be addressed in future publications in some detail.

\section{Some Vital Mathematical Prerequisites}

Based on his E-infinity Cantorian spacetime theory [1]-[21], it was recently argued by the author that the Casimir effect is a local manifestation of the quantum wave while dark energy is the global manifestation of the same [1]. The only difference is that of the details of the boundary conditions [1] [2]. It was further reasoned by the author that the universe as a whole has a one sided boundary akin to that of higher dimensional Möbius band and consequently the "local" Casimir effect ramifies at this one sided boundary located at infinity to produce the negative circular and expansive gravity pressure of the conjectured dark energy [1]-[3]. In other words, three rather mysterious physical notions are tied together and explained in terms of each other. At the top resides the quantum wave [4], which is not a mathematical artifact [4]-[8] but according to E-infinity theory of dark energy, a real physical entity [4] fully described by the empty set fixed by Connes-El Naschie bi dimension $\left(-1, \phi^{2}\right)$ where $\phi=1 /(\sqrt{5}+1)$ [1]-[8]. On the other hand the gradient caused by different wave energy density in different bounded regions of space compared to the unbounded outside of the same space is behind the Casimir forces which in the limit can be show to be equal to the difference between the quantum zero set $(0, \phi)$ and the wave empty set $\left(-1, \phi^{2}\right)$ leading to $\phi-\phi^{2}=\phi^{3}$ topological energy pressure or the reverse to get the negative sign, i.e. $\phi^{2}-\phi=-\phi^{3}$ [1] [5]-[8]. The situation is explained pictorially in Figure 1 and its legend. Finally at the edge of the universe there is only internal Casimir quantum wave pressure not balanced by outside pressure which is the dark energy concentration of 96 percent as per the consequences of Dvoretzky's theorem and the present author's dissection of Einstein's $E=m c^{2}$ to $E(O)=m c^{2} / 22$ for ordinary energy of the quantum particle and $E(D)=m c^{2}(21 / 22)$ for the dark energy of the quantum wave [7] [8]. Now for the Kerr geometry we realized that the power of topology is limited and that the subdivision of $E=m c^{2}(21 / 22)$ into $E=m c^{2}(5+16) / 22$ can only be a good approximation and cannot be written exactly in terms of fundamental sets as the zero set and the empty set.

The sources of the ideas contained in the present work go back to many years ago when we attempted to im- 
prove on the traditional fast and slow fission reactors using the modern mathematics of fractals and nonlinear dynamics [9]-[17]. The second source is our recent reinterpretation of Einstein's $E=m c^{2}$ and finally the third source is the unexpected results of the earlier mentioned Dvoretzky's theorem of Banach spaces [3] [6]. However in the final analysis building an actual reactor could not be possible, not even in principle, without first a sound theory [1]-[56] and second the combination of modern nanotechnology and state of the art Casimir effect experimentation [18]-[24]. In addition a reasonable amount of imaginative thinking similar to that of the man who is famed for inventing the $20^{\text {th }}$ century is also recommended [49] [57]. Armed with these mathematical and philosophical tools, the road from E-infinity via both lattice and branching polymer clusters [77]-[80] to a realistic model for a nano Casimir-dark energy spacetime reactor is not that long.

To keep the present paper short and yet to cover the large amount of the needed prerequisites we decided in favour of a condensed presentation coupled to a large number of references as mentioned earlier on in an apologetic way [1]-[88]. We recommend to start by reading Refs. [1], [2] and Ref. [60] as well as Ref. [74], and then it is personal choice as to how to proceed after that.

\section{The Boundary of the Holographic Boundary of the Universe and the Empty Set}

The holographic boundary theory goes back to the pioneering work of 'tHooft and Susskind [25]-[27]. On the other hand the principle that the boundary of a boundary is zero goes back to the out of the box thinking of J.A. Wheeler [28]. Pushing their ideas further still, it became obvious to the present author that the boundary of the holographic boundary is not only a zero limit set but actually a hierarchy of empty and emptier still sets quantified by von Neumann-Connes function and ramifying at a most general form of a one sided higher dimensional Möbius band [28]-[33]. This limit set resembles a fundamental polyhedron group or better still, a SchottkyKleinian group [29]-[33] which changes the topology of our conventional Casimir experiment to that of a sphere with internal Casimir pressure inflating the balloon-like universe and makes it expand into the surrounding "nothingness" fixed by the well known E-infinity formula $d_{C}^{(-\infty)}=\phi^{\infty}=0$ where $\phi=1 /(\sqrt{5}+1)$ [34]. From the preceding elementary reasoning it is clear that Casimir-effect and dark energy have the same cause, namely the topology of a Banach-spacetime like manifold and, as we repeatedly mentioned earlier on, the only difference is the difference of local exophysics and global endophysics and the respective associated boundary conditions [1] [2]. There is already a vast body of literature on the subject published in the last three years alone by the present author and his associates [1]-[56]. However what we are aiming at in the present paper is to point out the way to move from theory to useful, practical application of which nothing could be more important and pressing than building a free energy reactor, based on real science rather than wishful thinking. Thus we will combine the dreams of visionaries like N. Tesla with hardnosed modern mathematics and physics, which were not yet available in the time of Tesla [49].

\section{The Possibly Vital Role of Nanotechnology—Critical Dimensions}

There has been no want of imaginative experimental set ups for measuring, testing and visualizing the Casimir effect since it was proposed by Dutch physicist, H. Casimir [22]-[24]. In recent years nanotechnology invaded all scientific fields and played a significant role in Casimir effect experiments. Thanks to K-theory and E-infinity we now know that the true physical-mathematical connection between dark energy and ordinary measurable energy on the one side and the Casimir effect on the other side. A natural consequence of this discovered reality of the quantum wave, is rendering it a relatively simple task to find a way to harness dark energy or Casimir energy. Of course this "simple" is extremely difficult but no longer impossible. Never the less there are many speculative ideas about how to start, irrespective of the connection to Kerr black holes.

We can start for instance with a highly complex sub-structuring of space using nano tubes and nano particles and create that way fractal-like nanosphere packing modelling the moonshine conjecture which relates superstrings to other fields of theoretical physics. We stress in this connection that we have a clear model for our nano reactor based on two important facts. The first fact is the equivalence between branching polymer clusters and Cantorian-fractal spacetime [77]-[80]. The second is that we replace the Casimir plates of our model with Casimir spheres and then model these spheres with real nano particles. In principle this is our reactor. We thus presently have, in embryonic form, the main idea of constructing a nano universe and extracting dark energy from its nano boundary of its holographic boundary. Our program to actually extract energy from such a nano reactor 
may still need five or more years as we said earlier on but the road is marked and reasonably clear. It is only at the edge of the universe that $96 \%$ of the energy resides as dark energy as per Dvoretzky's theorem. However we could create many nano universes from which its $96 \%$ energy concentration could be extracted without actually reaching to the boundary of our universe which is of course factually impossible [3]-[8]. On the other hand if we could produce nano Kerr black holes, then a Penrose process could be feasible after all following broadly the preceding lines of speculation. In all events we must draw attention to the fact that in many fundamental problems in theoretical physics the equations of state becomes simplest at a critical point. Not only that but self similarity prevails at such criticality. This will actually be our road to branching polymers and our nano Casimir reactor.

\section{Laboratory Work between Real and Gedanken Experiments-Bethe Lattice (Figure 3) and Lattice Animals [77]-[80]}

In noncommutative geometry as well as E-infinity theory, the Penrose universe plays a significant role as a generic concrete model for both theories [50]-[53]. On the other hand Penrose universe or Penrose fractal tiling is basically a quasi-crystal mathematical model with the forbidden 5-fold symmetry [53] [54]. This form of matter not found naturally on earth, was produced experimentally by the great Israeli engineer D. Schechtman, who after facing a long period of fierce opposition from high profile scientists, for instance Nobel Laureate Linus Pauling, was rehabilitated and bestowed with a Nobel Prize. The 5 fold symmetry could be thought of theoretically as five Kaluza-Klein dimensions and using nano particles and nano tubes combinations we could build in the lab a nano holographic universe a la Penrose-'tHooft-Shechtman [5]-[8] akin to our own from which energy could be experimented with and extracted. For sure it will be a journey in unchartered seas with many trials and errors but sooner or later we will find out the right road to a Casimir energy nano reactor [1] [22]. There are other conceivable ways of producing artificial nano universes with high dimensionality for Dvoretzky's theorem to be applicable. For instance we could use Ji-Huan He's ten dimensional polytope [42] as a skeleton to grow on it a hierarchy of nano particles using the methods applied in the clustering of diffusion limited aggregation hinted at earlier on. In other words, we can let our scientific imagination run free but checked with E-infinity mathematical rigor and nanotechnological physical facts. We need not stop here because we are now very near indeed to a realizable nano Casimir reactor where we replace the Casimir plates by Casimir spheres. In turn our Casimir sphere could be taken to be nano particles and the entire model boils down to being branching polymer clusters (see Figure 3) which are frequently termed lattice animals with $D=4, D=6$ and $D=8$. This corresponds to SAW, percolation and proper lattice animals respectively [77]-[79].

\section{The Topological Casimir-Dark Energy Density Using E-Infinity Theory and the Correspondence with Quantum Field Theory}

It may come as a pleasant mild surprize that exact limits could easily be established for Casimir-dark energy using nothing more than the topology of our E-infinity Cantorian spacetime [56] [60]. We can do this in a variety of ways which are essentially tautologies leading to the same basic conclusion in the limit. Thus we could view the energy density of the space outside the two Casimir plates as that of Einstein's $E=m c^{2}$ density, i.e. $\gamma($ Einstein $)=1$. Inside the plate the energy density in the limit could only be a statical, quasi potential energy of the quantum particle, i.e. $E=m c^{2} / 22$ and consequently $\gamma(0)=1 / 22$. It follows then that the net pressure of the Casimir plates must be $1-(1 / 22)=21 / 22$ which is, in the meantime rather well known, as the dark energy density of spacetime. A second way to interpret the same situation and reach the same result is to argue that within the Casimir plates there is no "space" except for the empty set with a Hausdorff dimension $\phi^{2}$ where $\phi=1 /(\sqrt{5}+1)$. Outside on the other hand we have the zero set. As explained in some detail in Figure 1 , the difference is a net topological pressure $\phi-\phi^{2}=\phi^{3}$ which is the universal fluctuation of spacetime and simply the reciprocal value of its Hausdorff dimension $\left(1 / \phi^{3}\right)=4+\phi^{3}$ as discussed in earlier sections [3] [52] [56]. Finally we could see the situation as the difference of the completely empty set in the limit, i.e. zero between the Casimir plates and the spacetime fluctuation $\phi^{3}$ [60]. That way the Casimir effect could be set in the limit equal to $\phi^{3}$ and may easily be seen to be a relative to the Immirzi parameter $\phi^{6}$ and the Unruh temperature $\phi^{4}$ apart of Hardy's entanglement $\phi^{5}$, i.e. a member of a generalized quantum-topological entanglement 
family [60]. There is also one topological property of our quasi 4 dimensional spacetime which is so obvious and yet so terse that we often do not notice it, namely that intersection and union in this space are indistinguishable. This is so because of the deep elementary number theoretical fact that $2+2=(2)(2)=4$ for the topological dimension and $(1 / \phi)+(1 / \phi)^{2}=(1 / \phi)(1 / \phi)^{2}=4+\phi^{3}$ for the Hausdorff dimension. It is constructive at this point and for extensive later use to spell out in a nutshell the main correspondence between quantum field theory and E-infinity as far as the vacuum energy zero point is concerned. The vacuum state's lowest energy is identified by the condition $\hat{a}_{k}|0\rangle=0$. This corresponds to a von Neumann-Connes condition in Hausdorff rather than a topological evaluation which we could write symbolically as $|0\rangle=0 \rightarrow|0\rangle=\phi$. Similarly the energy between the Casimir plates changes from $\hat{a}|-1\rangle=-1$ and goes to $\phi^{2}$. That way the difference in energy that is the cause of the Casimir attraction can be written as

$$
\begin{aligned}
\Delta & =\hat{E}_{o}-E_{o} \\
& =\frac{1}{2}\left(\phi^{2}\right)-\frac{1}{2}(\phi) . \\
& =-\left(\phi^{3} / 2\right),
\end{aligned}
$$

The dimensional expression is of course $-\left(\phi^{3} / 2\right)\left(c \hbar / d^{2}\right)$ while for an electromagnetic field we have the well known result $-\left(\pi^{2} / 240\right)\left(c \hbar / d^{4}\right)$ [62].

\section{Derivation of Casimir Classical Result and a Short Comparative Study}

Disturbing the quantum vacuum with the objective of causing a shift in its energy density was an ingenious idea that came to Dutch physicist $\mathrm{H}$. Casimir when he was originally following a completely down to earth applied physics problem in the Philips Research and Development Laboratory where he was employed in 1948. Following again Figure 1 and designating the direction perpendicular to the Casimir $\mathrm{x}$ direction, then because of the boundary conditions which the electromagnetic field must satisfy on the plate, the wave vector takes only the discrete value $\pi n / d$ in the $x$ direction besides $K_{y}$ and $K_{z}$ in the two other directions. That way the energy between the plates will be changing to

$$
\sum_{n} \int \frac{\mathrm{d} K_{y} \mathrm{~d} K_{z}}{(2 \pi)^{2}} \sqrt{\left(\frac{\pi n}{d}\right)^{2}+k_{y}^{2}+k_{z}^{2}} .
$$

By varying the distance $d$ we must be keeping an eye on the variation of the energy density outside the two Casimir plates. However we can avoid this complex situation following A Zee by introducing the clever trio of an intermediate third plate. By holding the two outer plates 1 and 3 fixed and moving only the inner plate number 2, we can take L as large as we wish and come around the problem that way. Now two more simplifications: First we ignore polarization by regarding only a massless scaled field instead of an electromagnetic one. Second we reduce dimensionality of space to only two so that the integration over $K_{y}$ and $K_{z}$ drops out. That way the energy corresponding to our set up in Figure 1 would be

$$
E=F(d)+F(L-d)=\frac{\pi}{2 d} \sum_{n=1}^{\infty} n+F(L-d)
$$

where we have noted that the wave modes are given by $\sin (n \pi x / d)$ and $n=1, \cdots, \infty$ with energy equal $n \pi / d$. Now we face the most crucial problem which defines the real physics of quantum field theory and more generally quantum gravity, namely the divergence of the explosive term which physically should and in fact does not explode, namely

$$
\sum_{0}^{\infty} n
$$

It is the merit of E-infinity theory with its monadic random Cantor sets structure and the in built transfinite Turing computer and golden mean renormalization binary number system that these terms do not appear in the 
first place. However in 1948 Casimir had to deal with it in a different way mingling mathematics with physics as in the old pre 'tHooft-Veltman and Wilson dimensional regularization groups days. So while E-infinity proceeds in a purely mathematical way utilizing the result of pure mathematics regarding the continuum hypothesis and ultimate L [50] [53] [56] [60], we will proceed here in the old tradition of being eclectic and vacillate between mathematics and what may appear as physical common sense. This physical common sense leads to the introduction of a cut off term to make sure that only physically meaningful quantities are incorporated into our calculation. This term is

$$
\mathrm{e}^{(-a)(n \pi / d)}
$$

This cut off term could of course be justified on the physical grounds that the assumption of perfectly conducting Casimir plates breaks down at smaller wave lengths than atom dimensions. However the better argument is that spacetime is a Cantorian fractal. In fact 'tHooft's dimensional regularization is only possible in the author's opinion because 'tHooft is tacitly using a fractal spacetime in the indirect form of a Borel summation [1]-[8]. Taking a to be very small for instance of the order of the Bohr radius, then $1 / a$ becomes very large and one finds

$$
F(d)=\frac{d}{2 \pi a^{2}}-\frac{\pi}{24 d}+\text { higher order terms . }
$$

The Casimir force is then given by the negative value of the first derivation of $E$ with respect to $d$ where $d$ is much smaller than $L$ so that we find approximately the classical result for a massless scalar field

$$
\operatorname{Lim}_{a \rightarrow 0} \frac{\pi}{24}\left(\frac{1}{d^{2}}-\frac{1}{(L-d)^{2}}\right) \simeq \frac{\pi \hbar c}{24 d^{2}} .
$$

This extremely small force was measured with high accuracy experimentally and is real [63]-[66].

The preceding derivation must strike mathematical purists as a kind of voodoo and it may really be to a certain extent. In fact W. Pauli, with his superior intellect, sharp tongue and slightly over the top sense of humour, used the joke about this procedure by calling it theoretical physics circumcision. We cannot resolve this issue within the present paper nor a dozen more papers, however as a whole, we think that quantum gravity and theoretical physics is going in the general right direction of the ideas of Witten's topological quantum field theory as well as Grothendieck's conception of a monadic covering spacetime which harmonizes with E-infinity theory and is invading all of mathematical and theoretical physics in a steady pace. In the spirit of the above, we would like to show how some of the ideas and formalisms of E-infinity could be drawn in, albeit in a heuristic form to solve the problem at hand. Contemplating again Figure 1 we realize that by making $d \rightarrow 0$ we are de facto reducing spacetime to zero dimensions inside the Casimir plates. That would mean we have 3 dimensions outside the plates. However this is incorrect from an E-infinity viewpoint. The outside spacetime is 26 dimensional or more accurately $26+k$ dimensional where $k=\phi^{3}\left(1-\phi^{3}\right)$ is 'tHooft's renormalon and the 26 are those of Nambu-Veneziano bosonic strings space [50] [53]. The Casimir force pressuring the plates could thus be estimated by the ratio of $26+k$ to the total sum of all inverse coupling $\sum_{1}^{4} \bar{\alpha}_{i}=100$ divided by 2 [50] [53]. In other words we have

$$
\frac{1}{2} \frac{D \text { (bosonic strings) }}{\sum_{1}^{4} \bar{\alpha}_{i}}
$$

where $\bar{\alpha}_{1}=60, \bar{\alpha}_{2}=30, \bar{\alpha}_{3}=9, \bar{\alpha}_{4}=1$. Consequently we have

$$
\left(\frac{1}{2}\right)\left(\frac{26+k}{100}\right)=0.1309016995
$$

where our "classical" approximate result reported in the majority of the literature on the subject and given earlier on in this section is for a massless scalar field [64]

$$
\pi / 24=0.1308996939 \text {. }
$$


Another heuristic E-infinity way to look at the same problem is to take the ratio of the generators of $S U(2)$ of massive photons, i.e. the electroweak $W^{+}, W^{-}, Z^{\circ}$ and the total number of $S U(5)$ grand unification which is equivalent to a super symmetric standard model because $\operatorname{dim} S U(5)$ is twice that of $|S U(3) S U(2) U(1)|=12$. Consequently one finds

$$
\frac{|S U(2)|}{|S U(5)|}=\frac{3}{24}=0.125 \text {. }
$$

Again not a bad approximation to $\pi / 24$ of the classical value. This last solution is related to using the spin value $1+\phi^{3}$ introduced earlier on which converts $4+\phi^{3}$ to $5+\phi^{3}$ to convert $\phi^{3} / 2$ to that of the Unruh temperature, i.e. Casimir dynamical effect, namely $\left(\phi^{3}\right)\left(1+\phi^{3}\right)=\phi^{4} \quad$ [50] [53].

\section{On the Possibility of a Correspondence between a Rotating Kerr Black Hole and E-Infinity Conception to Ordinary and Dark Energy}

The following section is firmer than scientific speculation and a little less than a presentation of a definite model connecting a Kerr rotating black hole and its ergosphere to the quantum wave and quantum particle energy model of E-infinity theory [51] [52]. Let us call it for the moment just a rather strong new conjecture, namely that we could use the following to explain dark energy and dark matter and relate it all to the zero point vacuum fluctuation of quantum spacetime [1]-[8]. We recall our vital repeated assertion that our E-infinity model of dark energy relies on the dissection of $E=m c^{2}$ into $E(O)=m c^{2} / 22$ plus $E(D)=m c^{2}(21 / 22)$ where $E(O)$ is the ordinary measurable cosmic energy of the quantum particle modelled by a five dimensional zero set while $E(D)$ is the dark cosmic energy density of the quantum wave modelled by a five dimensional empty set [53] [56]. In particular $E(D)$ includes both dark energy and dark matter. Furthermore it turned out that the quantum wave empty set is the cobordism, i.e. the surface of the quantum particle [56] [60]. Thus we have only two "spacetime" monads as the basic structure of the E-infinity model, namely the zero set quantum particle surrounded by the empty set quantum wave [56]. We had to find further independent arguments to show that $E(D)=m c^{2}(21 / 22)$ which constitutes $96 \%$ of the total energy consists of $22 \%$ due to $26-4=22$ compactified bosonic dimensions [5], corresponds to dark matter density [1]-[8] while the rest $96-22=74 \%$ corresponds to repulsive pure dark energy [53] [55]. Further more the $96 \%$ is concentrated at the boundary of the universe by the celebrated Dvoretzky's theorem [6]. Now we come to our possibly new major insight based on the Kerr rotating black hole [70] [71].

In the case of Kerr singularity (see Figure 2) we have really three "spacetime" regions, unlike the simple static black hole because we have two event horizons and not only one [70] [71]. This is an inner event horizon surrounding the circular black hole pipe at the core and a second outer event horizon separating the ergosphere from the rest of the Kerr black hole [70] [71]. Our conjecture, which is based on several preliminary calculations, is that the ergosphere contains $22 \%$ of the total energy of the black hole and that this corresponds to the dark matter-like energy. In this sense we have a Kerr black hole nucleus having all the ordinary energy in it and that could be seen as a mini black hole model for elementary particles. Second we have the wave around the mini black hole elementary particle, which is two parts, namely the ergosphere with dark matter and the rest with pure dark energy. We have of course made use here of self similarity on a grand scale including the possibility that mini black holes are elementary particle while the universe as a whole could itself be a gigantic black hole. Clearly the present conjectured model still needs a great deal of elaboration, however if we are to succeed at making this model waterproof mathematically and physically consistent, it will be a major paradigm shift within our topological model of dark energy. It is not surprising that the geometry of Kerr spacetime gives us more details than E-infinity topology. This is natural because topologically a cow is a sphere but not geometrically. However our new equation for dark matter $E_{3}=m c^{2}(5 / 22)$ is only an approximation unlike our topological equation of E-infinity theory which is exact being based on zero and empty sets. The same applies for pure dark energy, $E_{4}=m c^{2}(16 / 22)$ which we stress is only an approximation and neither an exact irrational expression like $E(O)=\left(\phi^{5} / 2\right) m c^{2}$ nor an exact rational approximation like $E(O)=m c^{2} / 22$ nor like $E(D)=m c^{2}(21 / 22)$ 
(see Figure 2). We should remark here that the spacetime turbulence of Kerr black hole geometry resembles the Ivan Robins picture of Penrose twistors and visa versa as noted by the present author many years ago [72].

\section{Discussion, Conclusion and a Plea for a Peaceful Future}

The present work makes quite a leap from E-infinity dissection of Einstein's $E=m c^{2}$ into two, $E(O)=m c^{2} / 22$ plus $E(D)=m c^{2}(21 / 22)$ to connect our entire E-infinity scenario to the Kerr black hole and move from there to show that $E=m c^{2}(21 / 22)$ could be subdivided again into $E_{3}=m c^{2}(5 / 22)$ and $E_{4}=m c^{2}(16 / 22)$. Here $E_{3}$ is the dark matter density related to the ergosphere [70] [71] while the rest is pure dark energy. It would be a gross error to place the present nano reactor proposal within the context of science fiction. There is definitely a trivial element of speculation and trial and error in our grappling with mathematics, physics and common sense engineering towards a realistic design but that is all. Exploding stars and galaxies are scientific facts. Consequently to presume that these are only topological defects in to near infinitely large spacetime is not outlandish nor science fiction. The analogy between branching polymers and E-infinity space shown in Figure 3 testifies to the fact that we can simulate a mini spacetime universe and use it as a reactor [54]-[56]. In fact the near identity in origin of the Casimir effect and dark energy and the fact that both originate from the quantum wave aspect of quantum mechanics clearly shows to any open minded scientific thinker that to pursue clean free energy is not a scientific "crackpot" idea but a real and reachable aim. I ask the sensitive reader to forgive me for using the ugly word "crackpot", which is not a proper English word but merely slang which invaded the scientific English pseudo literature like a virus. The $4.5 \%$ of ordinary energy in the universe is nothing but the multiplicative volume of a five dimensional K-K zero set while the 95.5\% dark energy is the additive volume of the same 5D Kaluza-Klein empty set [34]. Seen that way we think that making humanity free from oil and traditional sources of energy is a higher and moral aim worth investing heavily in for what is a million or even billion dollar research grant funding compared to the three trillion dollar Iraq war [59]. In fact the highly enlightened rules of the United Arab Emirates are already looking towards a future free of oil based energy [61]. It was Nobel Laureate in Economics, Prof. J. Stiglitz who calculated with Prof. L. Bilmes the true cost of the Iraq war for the USA. The staggering three trillion dollars do not actually include the loss and destruction for the economy of the entire world. The author dares to say with a tongue in cheek, that the mere sight of only one trillion dollars funding for our nano Casimir-dark energy reactor is sufficient to make this reactor spontaneously pop out of spacetime like virtual particles! Some readers may rightly object that if this energy of spacetime is like dark energy then we cannot use it because of the wave collapse. However we can easily answer that it is sufficient to use the force of expansion of dark energy to produce a reactor. Besides, this energy is not dark energy, it is only like dark energy and Casimir energy does not collapse. In other words the door is wide open for a reactor. Apart of that, and without the need to play the devil's advocate, we know for a fact that a great deal of open and classified research is going on to develop wave collapse free measurement instruments that would bring dark energy of the real kind under control.

The author, who was born and raised in the Middle East with its unrivalled rich history and unparalleled chequered present day turbulent and logic defying politics feels morally obliged to call all the governments of the region to participate in a new dawn of science and dignified life for all living beings on this planet.

In conclusion of the conclusion we must stress the beauty of the theoretical grand picture reflected in Einstein's iconic equation $E=m c^{2}$ when being rewritten as

$E=m c^{2} / 22+m c^{2}(21 / 22)=m c^{2} / 22+m c^{2}(5 / 22)+m c^{2}(16 / 22)=m c^{2}$. The author would like to believe that Einstein would have loved it but then again this is probably an extremely immodest presumption which we will never know if it is true or not unless we can design a real spacetime nano reactor.

In concluding this conclusion it must be said that without the inspiration of Nobel Laureate G. Binnig via his remarkable book about fractals, nano technology and creativity [58] as well as his generous encouragement of the author, this paper and the Casimir reactor proposal would not have been possible. Needless to say, the work of Nobel Laureate G. 'tHooft was central to most of my work and the holographic principles of Susskind and 'tHooft is indispensible for the present work [86] [87]. Last but not least it was the seminal papers of G. Ord [88] that led me to my theory in the first place. All this is far of being a polite acknowledgement but is rather a statement of fact and a certificate of indebtedness. 


\section{References}

[1] El Naschie, M.S. (2015) Three Quantum Particles Hardy Entanglement from the Topology of Cantorian-Fractal Spacetime and the Casimir Effect as Dark Energy_A Great Opportunity for Nanotechnology. American Journal of Nano Research and Applications, 3, 1-5.

[2] El Naschie, M.S. (2014) Casimir-Like Energy as a Double Eigenvalue of Quantumly Entangled System Leading to the Missing Dark Energy Density of the Cosmos. International Journal of High Energy Physics, 1, 55-63.

[3] El Naschie, M.S. (2014) The Measure Concentration of Convex Geometry in a Quasi Banach Spacetime behind the Supposedly Missing Dark Energy of the Cosmos. American Journal of Astronomy \& Astrophysics, 2, 72-77.

[4] Slezak, M. (2015) Quantum Wave Function Gets Real. New Scientist, 225, 14. http://dx.doi.org/10.1016/S0262-4079(15)60242-1

[5] El Naschie, M.S. (2015) Dark Energy and Its Cosmic Density from Einstein’s Relativity and Gauge Fields Renormalization Leading to the Possibility of a New 'tHooft Quasi Particle. The Open Astronomy Journal, 8, 1-17. http://dx.doi.org/10.2174/1874381101508010001

[6] El Naschie, M.S. (2015) Banach Spacetime-Like Dvoretzky Volume Concentration as Cosmic Holographic Dark Energy. International Journal of High Energy Physics, 2, 13-21.

[7] El Naschie, M.S. (2014) From $E=m c^{2}$ to $E=m c^{2} / 22-$ A Short Account of the Most Famous Equation in Physics and Its Hidden Quantum Entanglement Origin. Journal of Quantum Information Science, 4, 284-291. http://dx.doi.org/10.4236/jqis.2014.44023

[8] El Naschie, M.S. (2014) The Hidden Quantum Entanglement Roots of $E=m c^{2}$ and Its Genesis to $E=m c^{2} / 22$ Plus $m c^{2}(21 / 22)$ Confirming Einstein's Mass-Energy Formula. American Journal of Electromagnetics and Applications, 2, 39-44. http://dx.doi.org/10.11648/j.ajea.20140205.11

[9] El Naschie, M.S. (1999) From Implosion to Fractal Spheres. A Brief Account of the Historical Development of Scientific Ideas Leading to the Trinity Test and Beyond. Chaos, Solitons \& Fractals, 10, 1955-1965. http://dx.doi.org/10.1016/S0960-0779(99)00030-2

[10] El Naschie, M.S. and Al Athel, S. (2000) Estimating the Eigenvalue of Fast Reactors and Cantorian Space. Chaos, Solitons \& Fractals, 11, 1957-1961. http://dx.doi.org/10.1016/S0960-0779(99)00069-7

[11] El Naschie, M.S. (2000) On Nishina's Estimate of the Critical Mass for Fussion and Early Nuclear Research in Japan. Chaos, Solitons \& Fractals, 11, 1809-1818. http://dx.doi.org/10.1016/S0960-0779(99)00172-1

[12] El Naschie, M.S. (2000) Remarks on Heisenberg's Farm-Hall Lecture on the Critical Mass of Fast Neutron Fission. Chaos, Solitons \& Fractals, 11, 1327-1333. http://dx.doi.org/10.1016/S0960-0779(99)00136-8

[13] El Naschie, M.S. and Hussein, A. (2000) On the Eigenvalue of Nuclear Reaction and Self-Weight Buckling. Chaos, Solitons \& Fractals, 11, 815-818. http://dx.doi.org/10.1016/S0960-0779(99)00106-X

[14] El Naschie, M.S. (2000) Elastic Buckling Loads and Fission Critical Mass as an Eigenvalue of a Symmetry Breaking Bifurcation. Chaos, Solitons \& Fractals, 11, 631-639. http://dx.doi.org/10.1016/S0960-0779(99)00063-6

[15] El Naschie, M.S. (2000) On the Zel'dovich-Khuriton Critical Mass for Fast Fission. Chaos, Solitons \& Fractals, 11, 819-824. http://dx.doi.org/10.1016/S0960-0779(99)00113-7

[16] El Naschie, M.S. (2000) On the Eigenvalue of Transport Reaction Involving Fast Neutrons. Chaos, Solitons \& Fractals, 11, 929-934. http://dx.doi.org/10.1016/S0960-0779(99)00066-1

[17] El Naschie, M.S. (2000) Heisenberg’s Critical Mass Calculations for an Explosive Nuclear Reaction. Chaos, Solitons \& Fractals, 11, 987-997. http://dx.doi.org/10.1016/S0960-0779(99)00110-1

[18] El Naschie, M.S. (1998) Chaos and Fractals in Nano and Quantum Technology. Chaos, Solitons \& Fractals, 9, 17931802.

[19] El Naschie, M.S. (2006) Nanotechnology for the Developing World. Chaos, Solitons \& Fractals, 30, 769-773. http://dx.doi.org/10.1016/j.chaos.2006.04.037

[20] El Naschie, M.S. (2007) The Political Economy of Nanotechnology and the Developing World. International Journal of Electrospun Nanofibrers and Applications, 1, 41-50.

[21] El Naschie, M.S. (1998) Some Tentative Proposals for the Experimental Verification of Cantorian Micro Spacetime. Chaos, Solitons \& Fractals, 9, 143-144. http://dx.doi.org/10.1016/S0960-0779(97)00175-6

[22] Johnston, H. (2012) Physicists Solve Casimir Conundrum. Physicsworld.com. 18 July 2012.

[23] Rencroft, S. and Swain, J. (1998) What Is the Casimir Effect? Scientific American, 22 June 1998.

[24] Wongjun, P. (2015) Casimir Dark Energy, Stabilization of the Extra Dimensions and Gauss-Bonnet Term. The Euro- 
pean Physical Journal C, 75, 6. http://dx.doi.org/10.1140/epjc/s10052-014-3237-0

[25] El Naschie, M.S. (2007) A Review of Applications and Results of E-Infinity Theory. International Journal of Nonlinear Sciences and Numerical Simulation, 8, 11-20. http://dx.doi.org/10.1515/IJNSNS.2007.8.1.11

[26] Smolin, L. (2001) The Strong and the Weak Holographic Principles. Nuclear Physics B, 601, $209-247$. http://dx.doi.org/10.1016/S0550-3213(01)00049-9

[27] El Naschie, M.S. (2006) Holographic Dimensional Reduction: Center Manifold Theorem and E-Infinity. Chaos, Solitons \& Fractals, 29, 816-822. http://dx.doi.org/10.1016/j.chaos.2006.01.013

[28] Misner, C., Thorne, K. and Wheeler, J.A. (1973) Gravitation. Freeman, New York.

[29] El Naschie, M.S. (2003) Kleinian Groups in E-Infinity and Their Connection to Particle Physics and Cosmology. Chaos, Solitons \& Fractals, 16, 637-649. http://dx.doi.org/10.1016/S0960-0779(02)00489-7

[30] El Naschie, M.S. (2005) A Guide to the Mathematics of E-Infinity Cantorian Spacetime Theory. Chaos, Solitons \& Fractals, 25, 955-964.

[31] El Naschie, M.S. (2004) The Concepts of E-Infinity: An Elementary Introduction to the Cantorian-Fractal Theory of Quantum Physics. Chaos, Solitons \& Fractals, 22, 495-511. http://dx.doi.org/10.1016/j.chaos.2004.02.028

[32] El Naschie, M.S. (2003) Complex Vacuum Fluctuation as a Chaotic "Limit” Set of Any Kleinian Group Transformation and the Mass Spectrum of High Energy Particle Physics via Spontaneous Self Organization. Chaos, Solitons \& Fractals, 17, 631-638. http://dx.doi.org/10.1016/S0960-0779(02)00630-6

[33] El Naschie, M.S. (2003) Modular Groups in Cantorian E-Infinity High Energy Physics. Chaos, Solitons \& Fractals, 16, 353-366. http://dx.doi.org/10.1016/S0960-0779(02)00440-X

[34] El Naschie, M.S. (1994) On Certain “Empty” Cantor Sets and Their Dimensions. Chaos, Solitons \& Fractals, 4, 293296. http://dx.doi.org/10.1016/0960-0779(94)90152-X

[35] He, J.H., Xu, L., Zhang, L.N. and Wu, X.H. (2007) Twenty-Six Dimensional Polytope and High Energy Spacetime Physics. Chaos, Solitons \& Fractals, 33, 5-13. http://dx.doi.org/10.1016/j.chaos.2006.10.048

[36] El Naschie, M.S. (1994) Is Quantum Space a Random Cantor Set with a Golden Mean Dimension at the Core? Chaos, Solitons \& Fractals, 4, 177-179. http://dx.doi.org/10.1016/0960-0779(94)90141-4

[37] El Naschie, M.S. (2008) Mathematical Foundation of E-Infinity via Coxeter and Reflection Groups. Chaos, Solitons \& Fractals, 37, 1267-1268. http://dx.doi.org/10.1016/j.chaos.2008.02.001

[38] El Naschie, M.S. (1995) Banach-Tarski Theorem and Cantorian Micro Spacetime. Chaos, Solitons \& Fractals, 5, 1503-1508. http://dx.doi.org/10.1016/0960-0779(95)00052-6

[39] El Naschie, M.S. (1995) On the Initial Singularity and the Banach-Tarski Theorem. Chaos, Solitons \& Fractals, 5, 1391-1392. http://dx.doi.org/10.1016/0960-0779(95)99645-2

[40] El Naschie, M.S. (1998) Cobe Satellite Measurement, Hyperspheres, Superstrings and the Dimension of Spacetime. Chaos, Solitons \& Fractals, 9, 1445-1471. http://dx.doi.org/10.1016/S0960-0779(98)00120-9

[41] El Naschie, M.S. (2001) Infinite Dimensional Branes and the E-Infinity Topology of Heterotic Superstrings. Chaos, Solitons \& Fractals, 12, 1047-1055. http://dx.doi.org/10.1016/S0960-0779(00)00130-2

[42] El Naschie, M.S. (2007) Ji-Huan He’s Ten Dimensional Polytope and High Energy Particle Physics. International Journal of Nonlinear Sciences \& Numerical Simulation, 8, 475-476. http://dx.doi.org/10.1515/IJNSNS.2007.8.4.475

[43] El Naschie, M.S. (1999) Hyperdimensional Geometry and the Nature of Physical Spacetime. Chaos, Solitons \& Fractals, 10, 155-158. http://dx.doi.org/10.1016/S0960-0779(98)00235-5

[44] Finkelstein, D. (1982) Quantum Sets and Clifford Algebras. International Journal of Theoretical Physics, 21, $489-503$. http://dx.doi.org/10.1007/BF02650180

[45] El Naschie, M.S. (2002) Derivation of the Threshold and Absolute Temperature $T_{c}=273.16 \mathrm{~K}$ from the Topology of Quantum Spacetime. Chaos, Solitons \& Fractals, 14, 1117-1120. http://dx.doi.org/10.1016/S0960-0779(02)00053-X

[46] El Naschie, M.S. (2008) Quarks Confinement via Kaluza-Klein Theory as a Topological Property of Quantum Classical Spacetime Phase Transition. Chaos, Solitons \& Fractals, 35, 825-829. http://dx.doi.org/10.1016/j.chaos.2007.08.057

[47] El Naschie, M.S. (2002) On a Class of General Theories for High Energy Particle Physics. Chaos, Solitons \& Fractals, 14, 649-668. http://dx.doi.org/10.1016/S0960-0779(02)00033-4

[48] He, J.H. (2009) Hilbert Cube Model for Fractal Spacetime. Chaos, Solitons \& Fractals, 42, 2754-2759. http://dx.doi.org/10.1016/j.chaos.2009.03.182

[49] Lomas, R. (1999) The Man Who Invented the Twentieth Century: Nicola Tesla, Forgotten Genius of Electricity. Head- 
line Books, London.

[50] Helal, M., Marek-Crnjac, L. and He, J.H. (2013) The Three Page Guide to the Most Important Results of M. S. El Naschie's Research in E-Infinity Quantum Physics and Cosmology. Open Journal of Microphysics, 3, 141-145. http://dx.doi.org/10.4236/ojm.2013.34020

[51] El Naschie, M.S. (2004) A Review of E-Infinity Theory and the Mass Spectrum of High Energy Particle Physics. Chaos, Solitons \& Fractals, 19, 209-236. http://dx.doi.org/10.1016/S0960-0779(03)00278-9

[52] El Naschie, M.S. (2009) The Theory of Cantorian Spacetime and High Energy Particle Physics (An Informal Review). Chaos, Solitons \& Fractals, 41, 2635-2646. http://dx.doi.org/10.1016/j.chaos.2008.09.059

[53] Marek-Crnjac, L. and He, J.H. (2013) An Invitation to El Naschie’s Theory of Cantorian Space-Time and Dark Energy. International Journal of Astronomy and Astrophysics, 3, 464-471. http://dx.doi.org/10.4236/ijaa.2013.34053

[54] El Naschie, M.S. (2013) A Resolution of Cosmic Dark Energy via a Quantum Entanglement Relativity Theory. Journal of Quantum Information Science, 3, 23-26. http://dx.doi.org/10.4236/jqis.2013.31006

[55] El Naschie, M.S. (2013) What Is the Missing Dark Energy in a Nutshell and the Hawking-Hartle Quantum Wave Collapse. International Journal of Astronomy \& Astrophysics, 3, 205-211. http://dx.doi.org/10.4236/ijaa.2013.33024

[56] El Naschie, M.S. (2013) Topological-Geometrical and Physical Interpretation of the Dark Energy of the Cosmos as a “Halo” Energy of the Schrodinger Quantum Wave. Journal of Modern Physics, 4, 591-596. http://dx.doi.org/10.4236/jmp.2013.45084

[57] Peat, F.D. (1983) In Search of Nikola Tesla. Ashgrove Publications, London \& Bath.

[58] Susskind, L. and Lindesay, J. (2005) The Holographic Universe. World Scientific, Singapore.

[59] Stiglitz, J. and Bilmes, L. (2008) The Three Trillion Dollar War: The True Cost of The Iraq Conflict. Allen-Lane, Penguin Books, London.

[60] El Naschie, M.S. (2013) A Unified Newtonian-Relativistic Quantum Resolution of Supposedly Missing Dark Energy of the Cosmos and the Constancy of the Speed of Light. International Journal of Modern Nonlinear Theory \& Application, 2, 43-54. http://dx.doi.org/10.4236/ijmnta.2013.21005

[61] Malek, C. (2015) Abu Dhabi Crown Prince Details UAE Leaders' Vision of Future without Oil. The National Newspaper, UAE, 10th February 2015.

http://www.thenational.ae/uae/government/abu-dhabi-crown-prince-details-uae-leaders-vision-of-future-without-oil?ut m_content='\%20vision\%20of\%20future\%20without\%20oil

[62] Zee, A. (2003) Quantum Field Theory in a Nutshell. Princeton University Press, Princeton.

[63] Duplantier, B. and Rivasseau, V., Eds. (2003) Vacuum Energy-Renormalization. Birkhauser, Basel.

[64] Milonni, P.W. (1994) The Quantum Vacuum. Academic Press, Boston.

[65] Parsegian, V.A. (2006) van der Waals Forces. Cambridge University Press, Cambridge.

[66] Huang, K. (2007) Fundamental Forces of Nature. World Scientific, Singapore. http://dx.doi.org/10.1142/6447

[67] Wapner, L.M. (2005) The Pea and the Sun. A.K. Peters Ltd., Wellesley.

[68] Auffray, J.P. (2015) E-Infinity, Zero Set, Absolute Space and the Photon Spin. Journal of Modern Physics, 6, 536-545. http://dx.doi.org/10.4236/jmp.2015.65058

[69] Connes, A. (1994) Noncommutative Geometry. Academic Press, San Diego. (See in particular pages 88-93 and Fig. II.4)

[70] Wald, R.M. (1984) General Relativity. The University of Chicago Press, Chicago and London. (See in particular page 319 and Fig. 12.6)

[71] Sternberg, S. (2012) Curvature in Mathematics and Physics. Dover Publications, New York.

[72] El Naschie, M.S. (2011) On Twistors in Cantorian E-Infinity Space. Chaos, Solitons \& Fractals, 12, 741-746. http://dx.doi.org/10.1016/S0960-0779(00)00193-4

[73] El Naschie, M.S. (2015) On a Casimir-Dark Energy Nano Reactor. American Journal of Nano Research and Application, 3, 12-16.

[74] Ho, M.W., El Naschie, M. and Vitiello, G. (2015) Is Spacetime Fractal and Quantum Coherent in the Golden Mean. Global Journal of Science Frontier Research-A: Physics and Space Science, 15, 61-80.

[75] El Naschie, M.S. (2015) From Kantian-Reinen Vernunft to the Real Dark Energy Density of the Cosmos via the Measure Concentration of Convex Geometry in Quasi Banach Spacetime. Open Journal of Philosophy, 5, 123-130. http://dx.doi.org/10.4236/ojpp.2015.51014

[76] El Naschie, M.S. (2015) Dark Energy and Its Cosmic Density from Einstein’s Relativity and Gauge Field Renormalization Leading to the Possibility of a New 'tHooft Quasi Particle. The Open Astronomy Journal, 8, 1-17. 
http://dx.doi.org/10.2174/1874381101508010001

[77] Stauffer, D. and Stanley, H.E. (1996) From Newton to Mandelbrot. 2nd Edition, Springer, Berlin.

[78] El Naschie, M.S. (1998) Branching Polymers and the Fractal Cantorian Spacetime. Chaos, Solitons \& Fractals, 9, 135-141. http://dx.doi.org/10.1016/S0960-0779(97)00133-1

[79] El Naschie, M.S. (1997) The Bethe Lattice and the Dimension of Micro Spacetime. Chaos, Solitons \& Fractals, 8 , 1887-1889. http://dx.doi.org/10.1016/S0960-0779(97)00130-6

[80] Miltao, M.S.R. (2008) Casimir Energy for a Double Spherical Shell: A Global Mode Sum Approach. Physical Review D, 78, Article ID: 065023. http://dx.doi.org/10.1103/PhysRevD.78.065023

[81] El Naschie, M.S. (2015) Computing Dark Energy and Ordinary Energy of the Cosmos as a Double Eigenvalue Problem. Journal of Modern Physics, 6, 348-395. http://dx.doi.org/10.4236/jmp.2015.64042

[82] El Naschie, M.S. (2015) A Fractal Rindler-Regge Triangulation in the Hyperbolic Plane and Cosmic de Sitter Accelerated Expansion. Journal of Quantum Information Science, 5, 24-31. http://dx.doi.org/10.4236/jqis.2015.51004

[83] El Naschie, M.S. (2015) The Casimir Topological Effect and a Proposal for a Casimir-Dark Energy Nano Reactor. World Journal of Nano Science and Engineering, 5, 26-33. http://dx.doi.org/10.4236/wjnse.2015.51004

[84] El Naschie, M.S. (2014) From Highly Structured E-Infinity Rings and Transfinite Maximally Symmetric Manifolds to the Dark Energy Density of the Cosmos. Advances in Pure Mathematics, 4, 641-648. http://dx.doi.org/10.4236/apm.2014.412073

[85] Binnig, G. (1990) Ausdem Nichits: Über die Kreativitat von Natur und Mensch. Piper Verlag, Munich, Germany.

[86] 'tHooft, G. (1997) In Search of the Ultimate Building Blocks. Cambridge University Press, Cambridge.

[87] Susskind, L. (2010) An Introduction to the Holographic Universe-Black Holes, Information and the String Theory Revolution. World Scientific, Singapore.

[88] McKeon, D.G. and Ord, G.N. (1992) Time Reversal in Stochastic Processes and Dirac Equation. Physics Review Letters, 69, 3-4. http://dx.doi.org/10.1103/PhysRevLett.69.3 